\title{
Recycling graphene from supercapacitor electrodes as reinforcing filler for epoxy resins
}

\author{
Guozhan Jiang,,$^{1^{*}}$ Stephen J Pickering ${ }^{1}$
}

Suggested running head: Jiang et al. Recycling graphene for reinforcing epoxy

\footnotetext{
${ }^{1}$ Division of Materials Mechanics and Structures, University of Nottingham, Nottingham NG7 2RD, UK *Corresponding author. Current address: School of Biology Chemistry and Forensic Science, University of Wolverhampton, Wolverhampton WV1 1LY, UK. email: guozhan.jiang@wlv.ac.uk; Telephone: +44 (0)1902 322366
} 


\begin{abstract}
A wet shredding process has been developed for recycling graphene from the electrodes of supercapacitors into polymer composites. At first, supercapacitors are cut open to expose the interior graphene based electrodes. The electrodes are heat-treated at $200^{\circ} \mathrm{C}$ to remove the contained solvent, and the heat treatment temperature can be further increased to remove the polymer binder, which binds the graphene on an aluminium foil current collector. After heat treatment, the electrodes are shredded in an epoxy resin to strip off the graphene and the graphene was subsequently dispersed using a high shear mixer. The dispersed graphene is used directly as reinforcing filler for the epoxy resin. A content of $0.40 \%(w t)$ of the recycled graphene resulted in a significant increase in both the tensile strength and elongation at break of the epoxy resin. Removal of the binder increases the reinforcing effect of the recycled graphene. However, a compromise can be made to leave the binder in the recycled graphene in order to avoid secondary pollution.
\end{abstract}

Keywords: supercapacitors, graphene, recycling, epoxy, composites 


\section{Introduction}

Graphene is an emerging high performance material with remarkable electronic and mechanical properties. These characteristics have exhibited the potential for development of advanced technologies in nano-electronics [1], photonics [2], biosensors [3] and high strength polymer composites [4]. Extensive research has been conducted for the basic behaviour of matter, mass production and device fabrication. We are expecting practical applications to be reported soon. Use of graphene in manufacturing supercapacitor electrodes is one of the main areas under intensive development in recent years $[5,6]$. Graphene based supercapacitor electrodes are manufactured by deposition of a thin layer of graphene on a metallic current collector. A small amount of polymer binder is used to make a strong cohesion between graphene particles. It is also used as an adhesion to the current collector. Liquid electrolyte (electrolyte salt dissolved in an organic solvent) is dispersed throughout the pores of the electrodes.

Along with the extensive fundamental and application research in graphene based supercapacitor, a viable recycling technology is necessary to achieve a sustainable process, since mass production of graphene is still expensive. Moreover, any electronic components are legislated to be recycled according to the WEEE Directive 2012/19/EU of the European Union. In this work, a recycling process was developed for graphene based supercapacitor electrodes.

For a successful recycling process, the end use of the recycled graphene should be considered first. Graphene for electronics is usually of high-quality to achieve outstanding electron mobility and conductivity for optimal properties. If recycled graphene will be used for electronic applications, it would thus be rather difficult and energy-consuming to meet the technical. A feasible application area is to use the recycled graphene for fabrication of polymer nanocomposites, which take the advantage of its nanosize to achieve superior mechanical properties, rather than the use of its electronic properties for electronic devices.

Graphene in supercapacitor electrodes is adhered to a current collector. Consequently, a key step for recycling graphene from the electrode is to strip off the graphene from the current collector. Once stripped off, re-dispersion of the graphene particles bound by polymer binder is necessary to obtain graphene nanoplatetlets. Considerable effort has been given so far for dispersing graphene in a liquid medium. Current strategies for dispersing graphene overly relies on sonication, which will be limiting 
in large volume applications [7]. Alternative approaches such as high shear mixing or ideally simple agitation are a priority [7]. High shear has been proved as an effective methods for dispersing grahene in viscous liquids [8]. Stripping of graphene from current collector for recycling graphene electrodes may also be realised by strong shear force via a viscous liquid flow field. Therefore, a combination of stripping and dispersion in a viscous liquid was proposed as being effective.

Shredding is a basic step for size reduction in recycling industry. If graphene based electrodes are shredded in a viscous liquid such as an epoxy resin by a high shear mixer, stripping of graphene from current collector and subsequently dispersing the stripped graphene may be realized in one step. The dispersed graphene will then enforce the epoxy resin. The proposed recycling approach is termed as wet shredding in this work. One reason for choosing epoxy resin is that it is a viscous liquid that can impart shear force for stripping and dispersion. Subsequently the recycled graphene is used as reinforcing filler for the epoxy resin. Another reason is that nanofillers have been shown to improve the interlaminar strength and crack-propagation prevention properties of carbon fibre reinforced epoxy resin composites [9-11]. Carbon fibre reinforced epoxy resin composites (CFRP) have been widely applied in aerospace, automobile, civil and marine structures where high performance and lightweight structures are essential [12]. However, as a laminated structure, delamination is the main limitation factor since CFRP laminates are susceptible to crack initiation and propagation along the laminar interfaces, causing severe reduction in inplane strength and stiffness and even catastrophic failure [13]. We previously used wet shredding technology to recycle activated carbon in a activated carbon based electrode, where water is used as the shredding medium [14].

The aim of this work is to develop the proposed wet shredding technology for stripping graphene off the metallic current collector and then use the recycled graphene insitu as reinforcing filler for the epoxy resin. The recycling methods may also be extended to recycle graphene in other devices.

\section{Experimental}

\subsection{Materials}

In this work, we conducted recycling of both the off-cuts of graphene electrodes and prototype supercapacitors based on the electrodes. The graphene electrodes and fresh 
graphene (XG Science) were provided by Fraunhofer IPA in Stuttgart. The prototype supercapacitors based on the graphene electrodes were provided by Maxwell (Switzerland), and these were subjected to charge and discharge test (a standard test for supercapacitors). The epoxy resin was Gurit Prime ${ }^{\mathrm{TM}}$ 20LV. Gurit Prime ${ }^{\mathrm{TM}}$ slow hardener (a mixture of amine curing agent including mainly 3-aminomethyl-3,5,5-trimethylcyclohexylamine and 2piperazin-1-ylethylamine) was used to cure the epoxy with a mass ratio to epoxy of 26:100. The curing schedule was 16 hours at $50^{\circ} \mathrm{C}$.

\subsection{Heat treatment}

Graphene based supercapacitors were cut open using a cutting knife to remove the casing, pins, rubber disc seal and plastic packaging in a fume cupboard. The electrodes collected from two flat supercapacitors $(1.40 \mathrm{~g})$ were then put into a $200 \mathrm{~mL}$ round-bottom flask. The flask was then heated to $200^{\circ} \mathrm{C}$ under nitrogen using an Electrothermal ${ }^{\mathrm{TM}}$ heating mantle. All the solvent acetonitrile contained in the electrodes was evaporated and collected via a condenser in 15 minutes. After the solvent was recycled, the electrode was removed from the flask and placed in a sample boat and then further heated to $600^{\circ} \mathrm{C}$ for 5 minutes in a Carbolite ${ }^{\mathrm{TM}}$ tube furnace to remove the binder and electrolyte in order to examine the effect of binder removal on the reinforcing effects of the recycled graphene. When the off-cuts of electrode were recycled, similar heat treatment was performed in order to make a comparison with the recycled graphene from the supercapacitors.

\subsection{In-situ recycling and composite fabrication}

The heat treated electrodes were then put into $130 \mathrm{~mL}(146.0 \mathrm{~g})$ of the epoxy resin (this is the minimum amount to fill the mould and the tubing attached to it for injection), and shredded using a Waring heavy duty blender (WSB50E) for 5 minutes with a speed of $18000 \mathrm{rpm}$. After shredding using the Warring blender in the epoxy resin, the suspension was further mixed using a Silverson high shear mixer (Model L5M) at $7500 \mathrm{rpm}$ for 15 minutes to disperse the graphene. The hardener $(38.0 \mathrm{~g})$ was then added to the dispersion according to the ratio of resin to hardener, and the mixture was degassed in a vacuum chamber until no bubbles were generated. The degassed mixture was filtered on a $0.5 \mathrm{~mm}$ diameter screen to remove the aluminium particles and then injected into a $200 \mathrm{~mm} \times 300$ $\mathrm{mm} \times 2 \mathrm{~mm}$ mould. The moulding was cured according to the curing schedule. The graphene left on the filtered aluminium particles was tested using ashing method. 


\subsection{Characterisation}

\subsubsection{Thermogravimetric analysis (TGA) of the electrodes}

The conditions for thermal treatment were determined using thermogravimetric analysis (TG). The TGA experiment was conducted using a Q600 Simultaneous DSC-TGA (TA Instruments). About $10 \mathrm{mg}$ of the electrode was cut into small pieces and then placed into a platinum crucible. The sample was heated from room temperature to $750^{\circ} \mathrm{C}$ under nitrogen gas flow with a heating rate of $20^{\circ} \mathrm{C} / \mathrm{min}$.

\subsubsection{Tensile testing of the fabricated composite}

The mechanical properties of the recycled graphene/epoxy composites were determined using uniaxial tensile testing according to BS EN ISO 527-2. The moulded composite plate was cut using an abrasive water-jet cutter into 5 test coupons with a gauge length of $60 \mathrm{~mm}$, a width of $10 \mathrm{~mm}$ and a thickness of $2 \mathrm{~mm}$, as shown in Figure 3 . The tensile testing was conducted on an Instron universal testing machine with a load cell of 5 $\mathrm{kN}$ and a rate of $1 \mathrm{~mm} / \mathrm{min}$. The strain was measured using digital image correlation.

\subsubsection{Determination of the graphene content in the composite}

Ashing method was used to determine the content of deposition layer (including graphene, binder and/or electrolyte) on the electrodes and on the recycled aluminium particles. About $1 \mathrm{~g}$ of the electrode or the recycled aluminium particles was placed in a crucible and then heated in a maffle furnace at $600^{\circ} \mathrm{C}$ in air for 1 hour. The weight before and after heating in the furnace was used to calculate the deposition layer content on the electrode or on the recycled aluminium particles.

The binder and electrolyte content in the electrode was determined by pyrolysis of the electrode at $600^{\circ} \mathrm{C}$ for 5 minutes in Carbolite ${ }^{\mathrm{TM}}$ tube furnace. The content of graphene in the fabricated composite was determined by subtracting the binder and electrolyte content from the deposition layer content.

\subsubsection{Scanning electron microscopy (SEM)}

A Philips XL30 facility was used to examine the effect of heat treatment on the deposition layer of the electrode and the uniformity of recycled graphene in the fabricated composite samples. For examination of the uniformity, the cross-section of the composite samples were polished for 2 hours in a Struers DAP-7 polishing machine using four 
increasingly fine sandpaper (European P320, P600, P1200 and P4000). Tungsten coated field emission gun was used for high resolution imaging to observe the dispersion of the recycled graphene.

\section{Results and discussion}

\subsection{TG analysis of graphene based supercapacitor electrodes}

In this work, a wet-shredding technology was used to recycle graphene based supercapacitor electrodes. The shredding of the electrode was conducted in an epoxy resin, where the graphene adhered on the metallic current collector is stripped off into the viscous epoxy resin and then use the mixture of graphene and epoxy resin is used to mould a composite material. A schematic diagram for the wet shredding process is shown in Figure

1. Electrodes are removed from the collected prototype supercapacitors (as shown in Figure 2a) by cutting open the packaging case, and then mixed with epoxy resin. High speed blenders are used to strip the graphene off and then disperse in the epoxy resins (Figure $2 d$ and f). The mixture of epoxy resin and recycled graphene is subsequently fabricated into polymer composites (Figure 2c) after filtering out the aluminium particles formed from the aluminium current collector (Figure $2 \mathrm{e}$ ). Figure $2 \mathrm{~b}$ shows a picture of the epoxy resin before and after mixing graphene.

The electrode in this work is composed of graphene mixed with electrolyte and binder deposited on aluminium collector. The objective is to recycle the solvent acetonitrile adsorbed in the graphene electrode and the graphene as reinforcing filler for epoxy resin.

To investigate the temperature for removing the adsorbed acetonitrile and other components contained in the graphene based electrode, TG analysis was used. The TG/DTG traces of the electrode containing acetonitrile solvent and electrolyte are shown in Figure 3. There are four peaks at around $50^{\circ} \mathrm{C}, 190^{\circ} \mathrm{C}, 475^{\circ} \mathrm{C}$ and $560^{\circ} \mathrm{C}$ in the TG traces.

The two peaks are obviously due to the adsorbed acetonitrile. When acetonitrile is adsorbed into graphene, one part is on the surface and one part will be in the pores of graphene. The surface adsorbed acetonitrile can be evaporated at much lower temperature. In the present work, the surface acetonitrile evaporated at around $50^{\circ} \mathrm{C}$, which is close to its boiling point. The acetonitrile adsorbed in the pores of graphene is quite hard to be removed due to diffusion control, which is consistent with other small molecules adsorbed in a porous material [15]. 
The binder is polyvinylidene difluoride, which has a decomposition temperature of around $470-500^{\circ} \mathrm{C}$ [14]. Hence, the peak at $475^{\circ} \mathrm{C}$ is attributed to the decomposition of the binder in the electrode. The electrolyte is tetraethyl ammonium tetrafluoroborate (TEABF 4$)$, which is reported to decompose at around $560^{\circ} \mathrm{C}[14,16]$.

According to the decomposition kinetics of acetonitrile in nitrogen, acetonitrile is stable at a temperature up to $300^{\circ} \mathrm{C}[14,17]$. Hence, acetonitrile can be recycled at a temperature up to $300^{\circ} \mathrm{C}$. Since $300^{\circ} \mathrm{C}$ is the decomposition temperature of acetonitrile, and all the acetonitrile, both adsorbed on the surface and adsorbed into the pores of graphene, can be removed at $190^{\circ} \mathrm{C}$, a temperature between $190^{\circ} \mathrm{C}$ and $300^{\circ} \mathrm{C}$ can be used for removal of the acetonitrile adsorbed in the graphene electrode. In this work, a temperature of $200^{\circ} \mathrm{C}$ was selected to evaporate acetonitrile from the electrodes. Obviously, higher evaporation temperature will reduce the time to remove all the acetonitrile in the electrode. The temperature for removing binder and electrolyte can also be selected based on the TG trace of the electrode. The binder has a decomposition temperature of $475^{\circ} \mathrm{C}$, and the electrolyte salt has a decomposition of $560^{\circ} \mathrm{C}$. Thus, a temperature of higher than $560^{\circ} \mathrm{C}$ should be used to remove both the binder and the electrolyte salt simultaneously. In this work, a temperature of $600^{\circ} \mathrm{C}$ was selected to remove both the binder and the electrolyte salt.

The effect of heat treatment at different temperatures on the morphology of the electrode is shown in Figure 4. In both the original electrode and after heat treatment at $200^{\circ} \mathrm{C}$, there are small fibre-shape connections over the cracks in the graphene layer, which may be due to the binder polymer. In preparation of the graphene based supercapacitor electrode, a spray coating method was used to deposit the GNPs on the aluminium foil current collector [18]. After drying, thermal stress may cause the layer of graphene on the aluminium collector to form some cracks, as clearly shown in Figure 5a. Some of fibrils are present between the gaps of the cracks. After heat treatment at $600^{\circ} \mathrm{C}$, the fibre-shape connection disappeared. Probably, the fibrils are polymer binder, which are removed by the heat treatment.

\subsection{Tensile properties of epoxy/recycled graphene composites}

After stripping the graphene off the aluminium current collector, the mixture of graphene and epoxy together with the aluminium particles was subjected to high speed 
shear mixing at $7500 \mathrm{rpm}$. Figure 5 shows the cross section of the fabricated composites for different time intervals. When the mixing time was 5 minutes, a large agglomeration was observed as shown in Figure 5a. It can thus be concluded that the agglomerate is graphene that was not dispersed well for a time of 5 minutes. For a shear mixing time of 15 minutes, agglomeration of graphene was not observed (Figure 5b).

The tensile properties of the moulded composites are shown in Table 1, with a comparison of the properties of neat epoxy and fresh graphene reinforced epoxy. When the graphene is not well dispersed, the tensile strength of the obtained composite is lower than that of epoxy resin, as shown in the first entry of Table 1 . When the graphene is well dispersed, the tensile properties are clearly enhanced.

As shown in Table 1, all the graphene reinforced epoxy and neat epoxy samples have a Young's modulus of approximately $3 \mathrm{GPa}$. This is because a $0.4 \%$ loading of graphene is too low to result in a change in stiffness. However, the strength has been enhanced by the incorporation of recycled graphene. The neat epoxy resin has a tensile strength of $54 \mathrm{MPa}$ and an elongation at break of $2 \%$. A $0.4 \%$ loading of fresh graphene results in $6 \%$ increase in tensile strength and $20 \%$ increase in elongation at break. When the electrodes were heat treated at a temperature below $200^{\circ} \mathrm{C}$, the reinforcing effect of the recycled graphene is similar to the fresh graphene. When the electrodes were heat treated at $600^{\circ} \mathrm{C}$, the recycled graphene results in a higher increase in tensile strength and elongation at break than that heat treated below $200^{\circ} \mathrm{C}$, which just removed the solvent. After heat treatment at $600^{\circ} \mathrm{C}$, the binder polymer was removed. This indicates that the binder has a slight influence in dispersing graphene during wet shredding.

Similar results have been reported elsewhere for the use of virgin graphene to reinforced epoxy resins when nanosized graphene is dispersed in epoxy resin uniformly. For example, Rafiee et al. [8] found that up to $40 \%$ and $53 \%$ increase in the tensile strength and fracture toughness respectively was achieved by only a small amount (0.1 wt.\%) graphene in graphene platelets (GP)/epoxy nanocomposites. Bao et al. [19] reported that a $2 \%$ loading of graphene in epoxy resin resulted in a $113 \%$ increase in storage modulus. It can be therefore indicative of the potential value of recycled graphene if properly dispersed in epoxy resin.

Since both tensile strength and fracture toughness increase in testing, this "nano effect" indicates that the recycled graphene is dispersed into nanoscale by the recycling 
process. In the present recycling experiment, the stripped off graphene was dispersed only after 15 minutes because there was a substantial rise in temperature due to the heat generated by shear. The increase in temperature resulted in a significant decrease in the viscosity of the epoxy resin, which led to inefficiency in dispersion effect by shear force. If the dispersing time is increased by suitable measures to maintain temperature, the reinforcing effect would increase. From the above discussion, further heat treatment is not necessary after removing the solvent acetonitrile. The recycled graphene can be used as effective reinforcing filler for epoxy resin.

\subsection{Pollution analysis}

In this work, we developed a recycling method for graphene based supercapacitor electrodes based on heat treatment and subsequent high speed shear mixing. The heat treatment is necessary in order to remove the organic solvent contained in the electrode material. For a mild heat treatment at $200^{\circ} \mathrm{C}$, organic solvent can be removed in a short time, but the electrolyte salt and polymer binder are not decomposed. The decomposition products of both the electrolyte and polymer binder are toxic and not easy to be treated. On the other hand, as shown in Table 1, the reinforcing effect of the recycled graphene is not much different at different heat treatment temperatures. The presence of binder and electrolyte salt has not caused significant reduction in the reinforcing effect of the graphene. Therefore, it can be proposed that removal of polymer binder and electrolyte be unnecessary in order to avoid secondary pollution. After removal of the organic solvent contained in the electrode, the graphene can be stripped off and dispersed in epoxy resin for fabrication of polymer composites. Furthermore, CFRP usually has an upper using temperature of less than $200^{\circ} \mathrm{C}$ due to the decomposition of epoxy resin. At this temperature, the electrolyte and polymer binder left are stable.

\section{Conclusions}

In this work, a wet shredding process was developed for recycling graphene from the graphene based supercapacitor electrodes, which combines stripping graphene from current collector and then dispersing the stripped graphene in one wet shredding step after heat treatment to remove the organic solvent contained within the electrode.

From TG analysis, the adsorbed acetonitrile are composed of two parts: surface acetonitrile and acetonitrile in the pores. The acetonitrile in the pores needs to be removed 
at higher temperature. The polymer binder and electrolyte salt needs to be removed at even higher temperatures.

When the solvent is removed, electrolyte salt and polymer binder are left in the electrode. However, the presence of these two components has little effect on the reinforcing effect of the recycled graphene. Consequently, in order to avoid secondary pollution during recycling, these two components can be left in the composites.

\section{Acknowledgements}

The research leading to these results has received funding from the European Union Seventh Framework Programme (FP7/2007-2013) under grant agreement n²66391.

\section{References}

1. Freitag, M.: Graphene: nanoelectronics goes flat. Nature Nanotechnology. 3, 455-457 (2008).

2. Bonaccorso, F., Sun, Z., Hasan, T., and Ferrari, A.C.: Graphene photonics and optoelectronics. Nature Photonics. 4, 611-622 (2010).

3. Kuila, T., Bose, S., Khanra, P., Mishra, A.K., Kim, N.H., and Lee, J.H.: Recent advances in graphene-based biosensors. Biosensors and Bioelectronics. 26, 4637-4648 (2011).

4. Mittal, G., Dhand, V., Rhee, K.Y., Park, S.-J., and Lee, W.R.: A review on carbon nanotubes and graphene as fillers in reinforced polymer nanocomposites. Journal of Industrial and Engineering Chemistry. 21, 11-25 (2015).

5. Liu, C., Yu, Z., Neff, D., Zhamu, A., and Jang, B.Z.: Graphene-based supercapacitor with an ultrahigh energy density. Nano Letters. 10, 4863-4868 (2010).

6. Ke, Q. and Wang, J.: Graphene-based materials for supercapacitor electrodes - A review Journal of Materiomics. 2, 37-54 (2016).

7. Johnson, D.W., Dobson, B.P., and Coleman, K.S.: A manufacturing perspective on graphene dispersions. Current Opinion in Colloid \& Interface Science. 20, 367-382 (2015).

8. Rafiee, M.A., Rafiee, J., Wang, Z., Song, H., Yu, Z., and Koratkar, N.: Enhanced mechanial properties of nanocomposites at low graphene content. ACS nano. 3, 3884-3890 (2009).

9. Chatterjee, S., Wang, J.W., Kuo, W.S., Tai, N.H., Salzmann, C., Li, W.L., Hollertz, R., Nuesch, F.A., and Chu, B.T.T.: Mechanical reinforcement and thermal conductivity in expanded graphene nanoplatelets reinforced epoxy composites. Chemical Physics Letters. 531, 6-10 (2012).

10. Li, C., Browning, A.R., Christensen, S., and Strachan, A.: Atomistic simulations on multilayer graphene reinforced epoxy composites. Composites Part A. 43, 1293-1300 (2012).

11. Ning, H., Li, J.H., Hu, N., Yan, C., Liu, Y., Wu, L., Liu, F., and ZHang, J.: Interlaminar mechanical properties of carbon fiber reinforced plastic laminates modified with graphene oxide interleaf. Carbon. 91, 224-233 (2015).

12. Njuguna, J., Pielichowski, K., and Alcock, J.R.: Epoxy-based fibre reinforced nanocomposites. Advanced Engineering Materials. 9, 835-847 (2007). 
13. Sekine, H., Hu, N., and Kouchakzadeh, M.A.: Buckling analysis of elliptically delaminated composite laminates with consideration of partial closure of delamination. Journal of Composite Materials. 34, 551-574 (2000).

14. Jiang, G. and Pickering, S.J.: Recycling supercapacitors based on shredding and mild thermal treatment. Waste Management. 48, 465-470 (2016).

15. Suzuki, M., Misic, D.M., Koyama, O., and Kawazoe, K.: Study of thermal regeneration of spent activated carbons: thermaogravimetric measurement of various single component organics loaded on activated carbons. Chemical Engineering Science. 33, 271-279 (1978).

16. Prasad, M.R.R. and Sudhakarbabu, K.: Thermal decomposition of tetraethyl ammonium tetrafluoroborate. Journal of Thermal Analysis and Calorimetry. 115, 1901-1905 (2014).

17. Asmus, T.W. and Houser, T.J.: Pyrolysis kinetics of acetonitrile. Journal of Physical Chemistry. 73, 2555-2558 (1969).

18. Pullini, D., Siong, V., Tamvakos, D., Ortega, B.L., Sgroi, M.F., Veca, A., Glanz, C., Kolaric, I., and Pruna, A.: Enhancing the capacitance and active surface utilization of supercapacitor electrode by graphene nanoplatelets. Composites Science and Technology. 112, 16-21 (2015).

19. Bao, C., Guo, Y., Song, L., Kan, Y., Qian, X., and Hu, Y.: In situ preparation of functionalized graphene oxide/epoxy nanocomposites with effective reinforcements. Journal of Materials Chemistry. 21, 13290-13298 (2011). 


\section{List of Figures}

Figure 1. A schematic diagram for recycling graphene based supercapacitor electrodes as reinforcing fillers for epoxy resin.

Figure 2. An image of the prototype graphene based supercapacitor, its recycling process and moulded test coupons. The size of the supercapacitor had a size of $30 \times 30 \mathrm{~mm}$.

Figure 3. Thermmogravimetric (TG) trace of the graphene based supercapacitor electrode and its first derivative (DTG) to indicate the characteristic peaks.

Figure 4. SEM images of the graphene based supercapacitor electrodes before and after heat treatment at different temperatures (a) before heat treatment; (b) after heat-treated at $200^{\circ} \mathrm{C} ;(\mathrm{c})$ after heat-treated at $600^{\circ} \mathrm{C}$.

Figure 5. SEM images of the cross-sections of the fabricated composites using the recycled graphene. (a) The mixing time was 5 minutes after heat treatment at $200^{\circ} \mathrm{C}$; (b) The mixing time was 15 minutes after heat treatment at $200^{\circ} \mathrm{C}$.

\section{List of Tables}

Table 1. Mechanical properties of composites fabricated using epoxy resin (EP) and graphene recycled from electrode offcuts (ED) and supercapacitors (SC) at different temperatures indicated by Txxx, and different mixing time indicated by $\mathrm{M} \times \mathrm{min}$. The content of graphene is $0.40 \% \mathrm{wt}$ in the composites. 

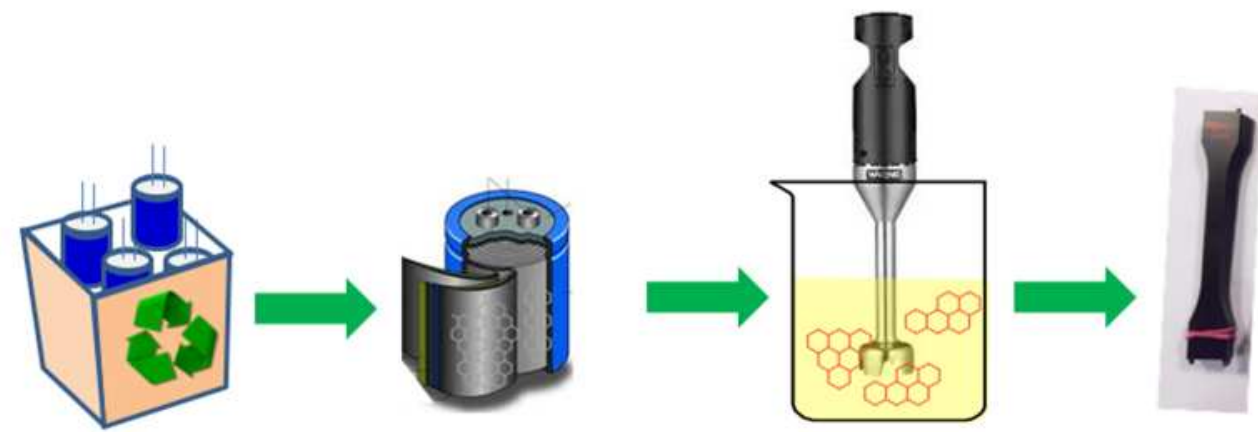

Figure 1 

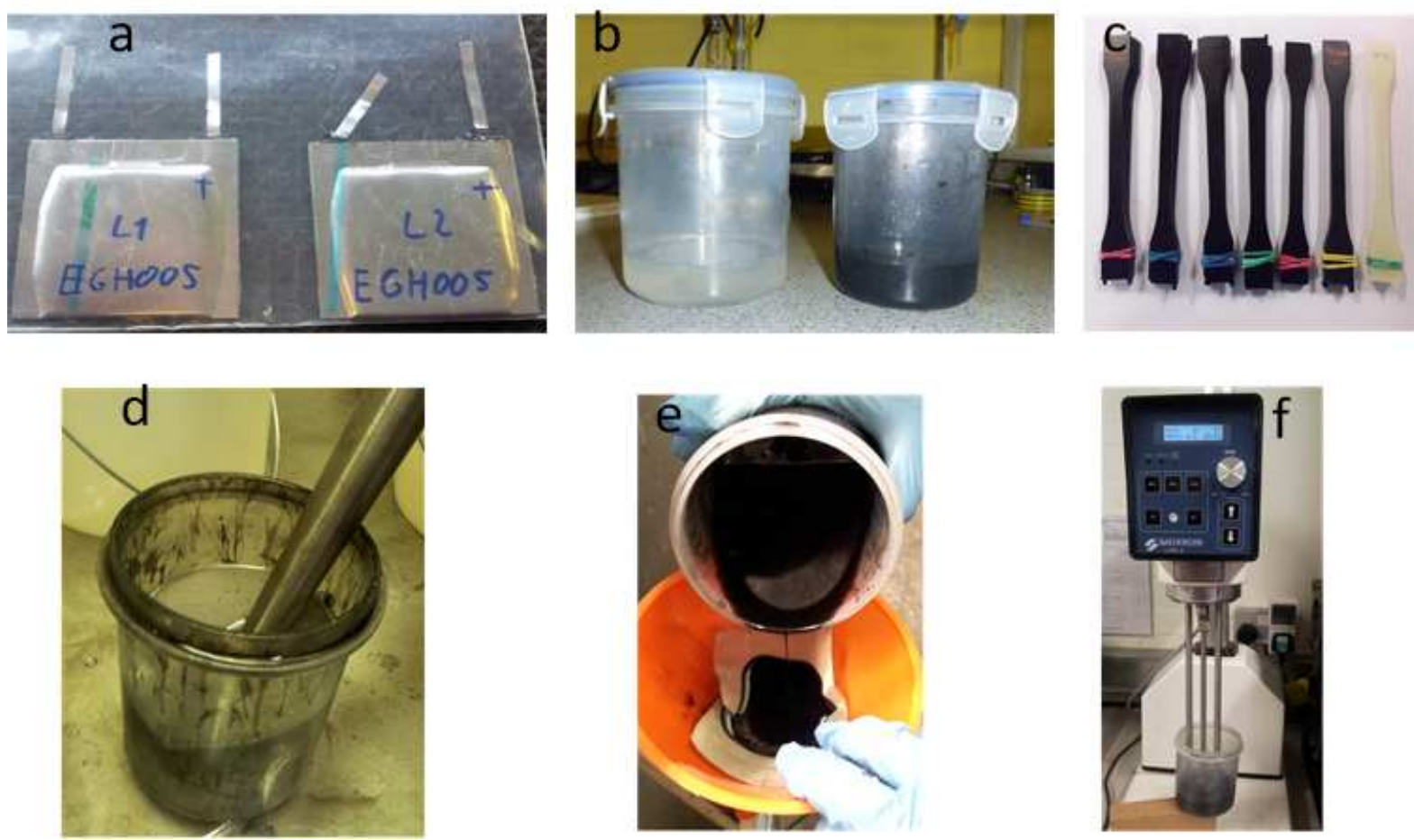

Figure 2

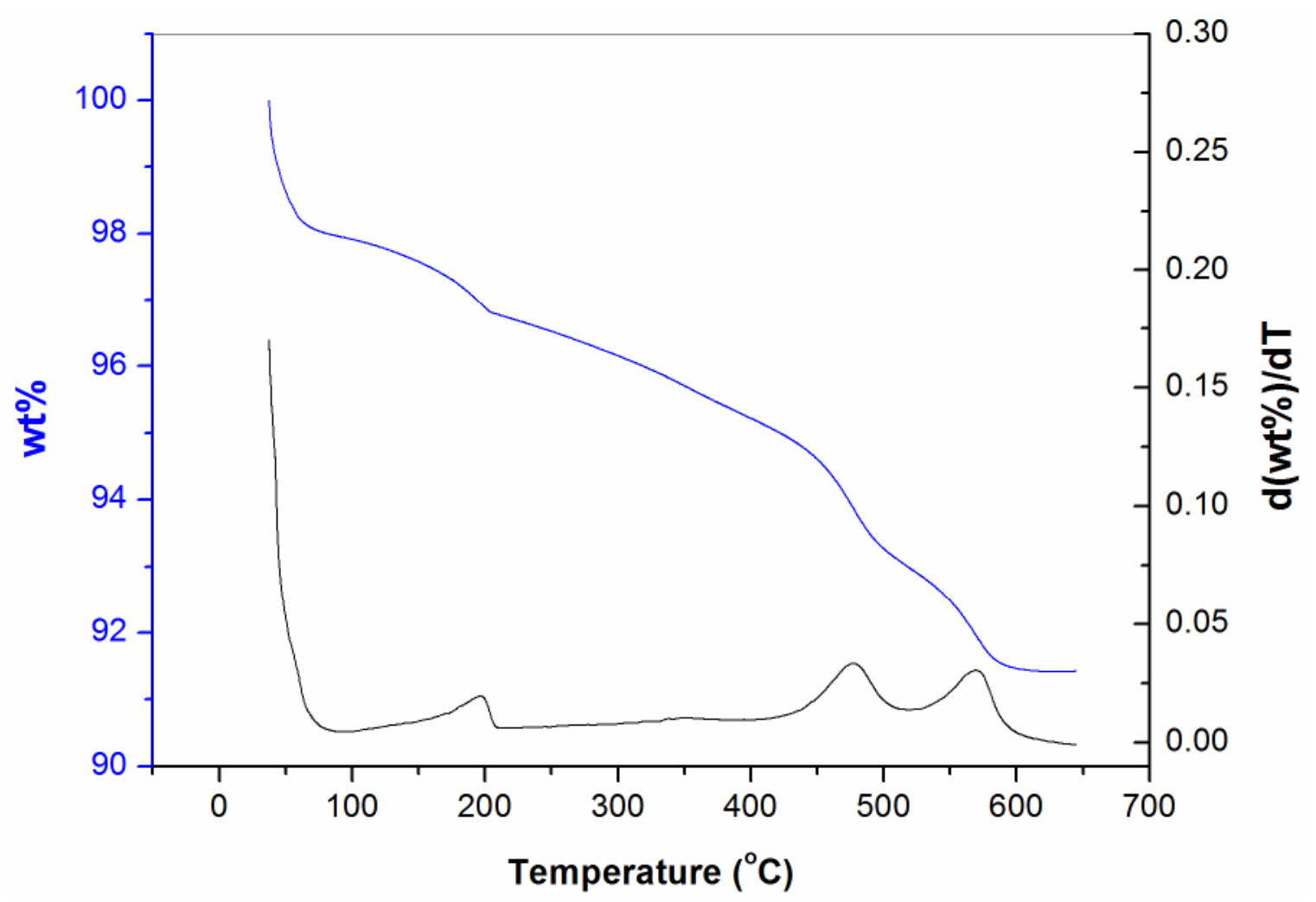

Figure 3 


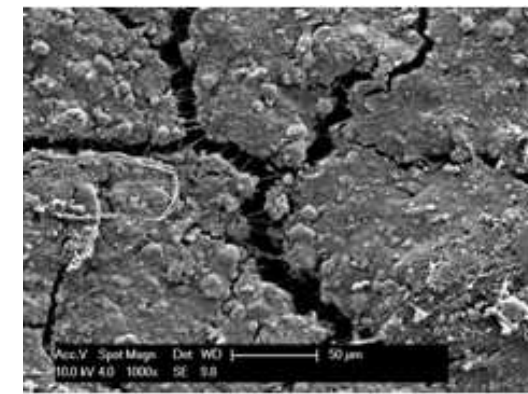

a

Figure 4

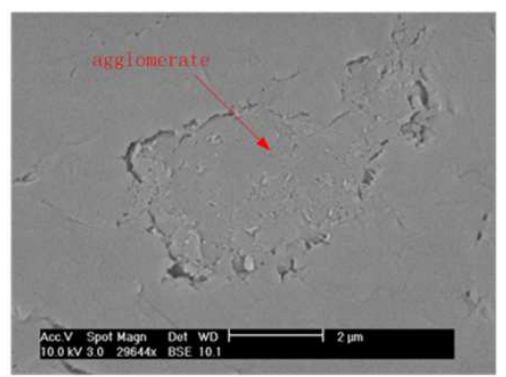

a

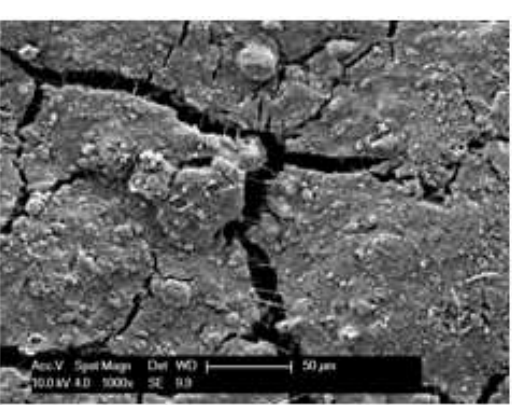

b

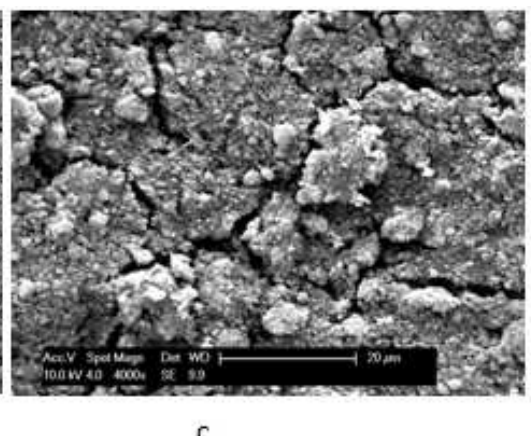

c

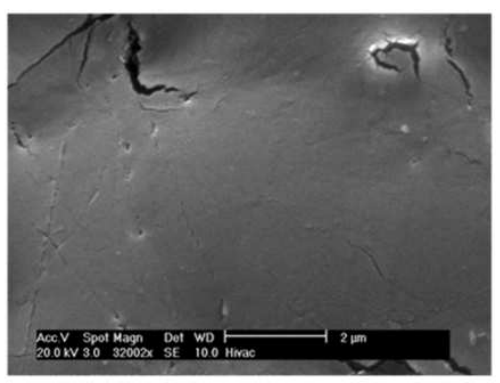

Figure 5 
Table 1. Mechanical properties of the composites fabricated using recycled graphene in epoxy resin. The content of graphene is $0.4 \%$ (wt) in the composites. In the first columnof the table, the meanings of the entries are as follows. EP: epoxy resin; ED: graphene recycled from electrode offcuts; EC: graphene recycled from supercapacitors (SC); T and the number: Heat treatment temperature.

\begin{tabular}{llll}
\hline Sample & $\begin{array}{l}\text { Tensile strength } \\
(\mathbf{M P a})\end{array}$ & $\begin{array}{l}\text { Young's } \\
\text { modulus }(\mathbf{G P a})\end{array}$ & $\begin{array}{l}\text { Elongation at } \\
\text { break }(\%)\end{array}$ \\
\hline EP & $54.20 \pm 1.83$ & $2.92 \pm 0.26$ & $2.09 \pm 0.20$ \\
EP/new graphene & $60.71 \pm 2.32$ & $2.89 \pm 0.077$ & $2.43 \pm 0.18$ \\
EP/ED-no heat & $58.83 \pm 1.85$ & $2.91 \pm 0.073$ & $2.09 \pm 0.20$ \\
treatment & & & \\
EP/ED-T200 & $56.56 \pm 1.89$ & $2.98 \pm 0.090$ & $2.16 \pm 0.053$ \\
EP/ED-T600 & $60.61 \pm 2.52$ & $3.17 \pm 0.092$ & $2.99 \pm 0.34$ \\
EP/SC-T600 & $61.17 \pm 1.93$ & $2.91 \pm 0.11$ & $2.77 \pm 0.20$ \\
\hline
\end{tabular}

